\title{
Control of sawteeth and triggering of NTMs with ion cyclotron resonance frequency waves in JET
}

\author{
E. Westerhof ${ }^{1}$, O. Sauter ${ }^{2}$, M.L. Mayoral ${ }^{3}$, D.F. Howell ${ }^{3}$, \\ M.J. Mantsinen ${ }^{4}$, M.F.F. Nave ${ }^{5}$, B. Alper ${ }^{3}$, C. Angioni ${ }^{2}$, P. Belo ${ }^{5}$, \\ R.J. Buttery ${ }^{3}$, A. Gondhalekar ${ }^{3}$, T. Hellsten ${ }^{6}$, T.C. Hender ${ }^{3}$, \\ T. Johnson ${ }^{7}$, P. Lamalle ${ }^{6,8}$, M.E. Maraschek ${ }^{9}$, K.G. McClements ${ }^{3}$, \\ F. Nguyen ${ }^{10}$, A.L. Pécquet ${ }^{10}$, S. Podda ${ }^{11}$, J. Rapp ${ }^{12}$, S.E. Sharapov ${ }^{3}$, \\ M. Zabiego ${ }^{10}$ and contributors to the EFDA JET \\ Workprogramme ${ }^{\mathrm{a}}$

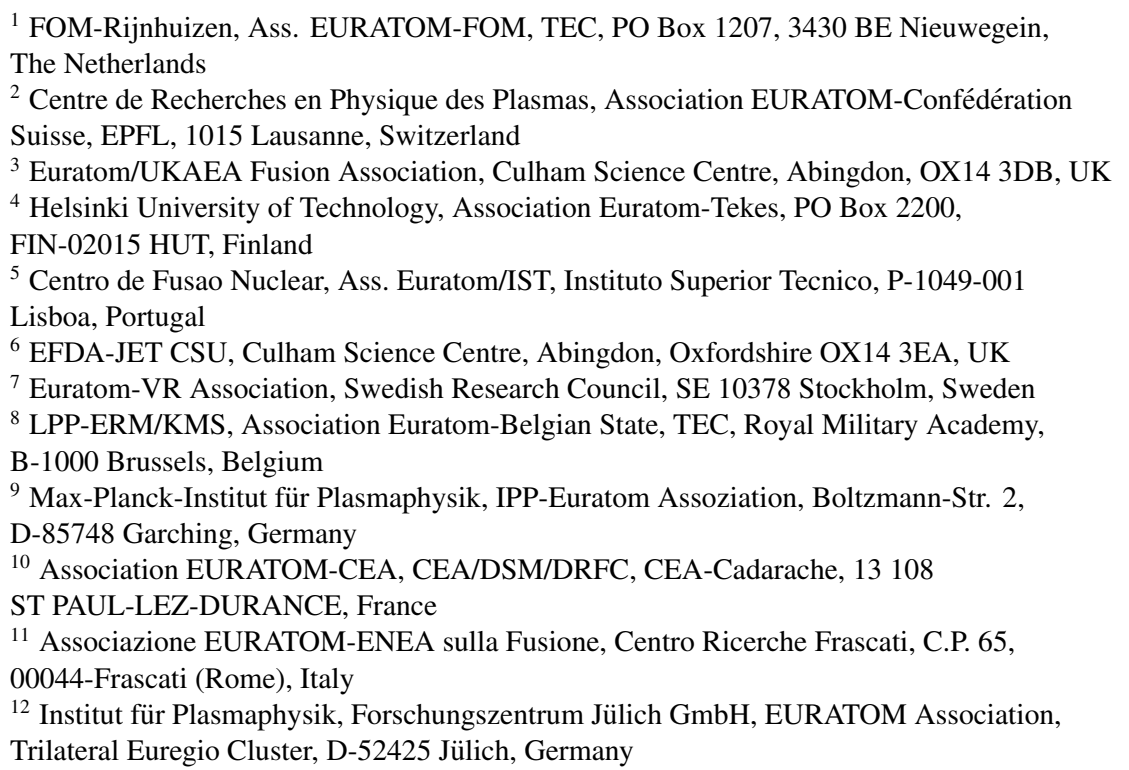

Received 11 March 2002, accepted for publication 4 September 2002

Published 9 October 2002

Online at stacks.iop.org/NF/42/1324

\begin{abstract}
A new scenario to delay or prevent neoclassical tearing mode (NTM) onset is presented. By active sawtooth destabilization, short period and low amplitude sawteeth are generated, such that the sawtooth produced NTM seed island is reduced and the threshold normalized plasma pressure for triggering of NTMs, $\beta_{\text {Nonset }}$, is increased. The scenario has been explored experimentally in the Joint European Torus (JET). Ion cyclotron resonance frequency (ICRF) waves tuned to the 2 nd harmonic $\mathrm{H}$-minority resonance have been used for sawtooth control. Whereas ICRF waves generally induce sawtooth stabilization, favouring the triggering of NTMs and reducing $\beta_{\text {Nonset }}$, the present experiments show that by toroidally directed waves, ion cyclotron current drive is produced, and that sawteeth can be destabilized by careful positioning of the 2 nd harmonic $\mathrm{H}$ resonance layer with respect to the sawtooth inversion radius. As a result, NTM onset is delayed and $\beta_{\text {Nonset }}$ is increased above its value obtained in discharges with additional heating from neutral beam injection alone.
\end{abstract}

PACS numbers: 52.55.Fc, 52.55.Tn, 52.55.Wq

\footnotetext{
a See annex of Pamela J. et al 2001 Overview of recent JET results and future perspectives Proc. Int. Conf. on Fusion Energy (Sorrento, 2000) (Vienna: IAEA).
} 


\section{Introduction}

In recent years, it has been found that in long pulse tokamak discharges, the achievable plasma pressure $p$ or beta, $\beta \equiv$ $p /\left(B_{0}^{2} / 2 \mu_{0}\right)$ where $B_{0}$ is the vacuum toroidal magnetic field in the plasma centre, is often limited to values below the limits predicted by ideal magnetohydrodynamics (MHD) [1-6]. The modes responsible for this limitation have been identified as resistive tearing modes, which form magnetic islands around resonant surfaces with low order rational values of the safety factor $q$, in particular around the $q=m / n=3 / 2$ and $q=2 / 1$ surfaces, where $m$ and $n$ are the poloidal and toroidal mode numbers, respectively. Although, these tearing modes are generally found to be linearly stable, once a sufficiently large seed island is formed and flattens the pressure profile across the magnetic island, the resultant perturbation to the neoclassical bootstrap current can result in a further growth of the mode [2,3]. Hence, these instabilities are called neoclassical tearing modes (NTMs). A key feature of these NTMs is the requirement for some trigger mechanism to create the necessary seed island. Usually a sawtooth crash or precursor provides this trigger, but it has also been seen to be provided by other perturbations like fishbones or edge localized modes (ELMs).

As NTMs are resistive modes, they grow on a relatively slow timescale and, with the exception of the $m=2, n=1$ NTM or $m=3, n=2 \mathrm{NTM}$ at low $q_{95}$, do not lead to a disruptive termination of the discharge. Rather, their main detrimental effect lies in the rapid transport across the magnetic islands which can grow to a sizable fraction of the tokamak minor radius [2]. This reduces significantly the maximum attainable central temperature and density (with a resultant confinement degradation of typically 15\%). As the $q=3 / 2$ surface usually lies in the middle of the confinement region supporting the pressure gradient, a key role is being played by the $m=3, n=2$ NTM. In a number of tokamaks the normalized plasma beta, $\beta_{\mathrm{N}}$, at which the $m=3, n=2 \mathrm{NTM}$ is triggered, is found to scale almost linearly with the normalized ion Larmor radius $\rho_{*}[7,5]$. Here, the normalized plasma beta is defined as $\beta_{\mathrm{N}} \equiv \beta[\%] a[\mathrm{~m}] B_{0}[\mathrm{~T}] / I_{\mathrm{p}}[\mathrm{MA}]$ where $a$ is the minor radius, and $I_{\mathrm{p}}$ the plasma current, and the normalized Larmor radius by $\rho_{*} \equiv \rho_{\mathrm{i}} / a$, where $\rho_{\mathrm{i}}$ is the thermal ion Larmor radius. When extrapolated to a reactor scale tokamak like the International Thermonuclear Experimental Reactor (ITER) [8] this would predict the destabilization of a $m=3, n=2$ NTM below the standard operating value. From a more detailed analysis of the data the opposite prediction has been obtained, namely, that the $m=3, n=2$ NTM would be stable in ITER [9]. This prediction is based on separate scalings for the sawtooth generated seed island size and the critical seed island size required for mode growth, and depends critically on the relative scaling of these two island sizes. Moreover, the extrapolation to ITER relies on reproducibility of the present experimental regime, standard ELMy H-mode with neutral beam injection (NBI) heating. In some JET experiments with significant ion cyclotron resonance frequency (ICRF) heating, the $m=3$, $n=2 \mathrm{NTM}$ is found to be triggered at very low $\beta_{\mathrm{N}}$ values, $\beta_{\mathrm{N}}<1$ or $\approx 1$, compared to those found generally with NBI heating [10]. This is most likely caused by large sawteeth, which are the result of sawtooth stabilization due to a high fast ion pressure in the centre, and similarly large NTM seeds. The triggering of NTMs at such low $\beta_{\mathrm{N}}$ values means that, in practice, JET is meta-stable to NTMs when in H-mode [11]. These observations clearly point to the benefits to be gained from control of the seeding perturbations, and to the necessity to better understand the seeding process [12-14].

In a reactor like ITER the energetic $\alpha$ particles produced by Deuterium-Tritium fusion are expected to cause sawtooth stabilization, which can result in monster sawtooth collapses. The triggering of NTM thus appears to be quite likely, and the development of a strategy for stabilization, or prevention, is prudent. Several strategies to stabilize NTMs have been proposed and applied successfully in different tokamaks. Noninductive current drive, for example by electron cyclotron current drive (ECCD), inside the magnetic islands has been proposed to stabilize tearing modes [15]. This way, one locally substitutes the missing bootstrap current inside the magnetic island and thus removes the unstable drive of NTMs $[16,17]$. ECCD has been used successfully in ASDEX-Upgrade [18], DIII-D [19], and JT60-U [20] to stabilize the $m=3, n=2$ NTM. Local current profile control, by either lower hybrid current drive (LHCD) or ECCD, can be used to improve the linear stability with respect to tearing modes [21] and thereby improve the resistance towards NTMs [22]. In COMPASS-D a $m=2, n=1 \mathrm{NTM}$, was suppressed by profile control through LHCD [23]. More recently, non-resonant magnetic perturbations have been suggested as a means to reduce the pressure perturbation across the island and the consequent drive of the NTMs [24].

Here, an alternative strategy is pursued in order to postpone the triggering of NTMs to higher $\beta_{\mathrm{N}}$ or to avoid their triggering. The obvious way to do this is by controlling the seed island generation. As in the majority of cases the latter is associated with the sawtooth, one way to achieve this is by sawtooth control. Sawteeth can be avoided by using scenarios with $q>1$. This has been studied on DIII-D [25]. However, $q>1$ can often only be maintained transiently, and in a reactor sawteeth also have the beneficial effect of removing the He-ash from the centre of the discharge. Thus, the proposed scheme for the prevention of NTMs consists of sawtooth destabilization resulting in more frequent, lower amplitude sawtooth crashes, which will be associated with smaller magnetic perturbations and, consequently, will lead to the generation of smaller seed islands. This means that higher $\beta_{\mathrm{N}}$ will be reached before a NTM is triggered or that the triggering of NTMs will be avoided. The necessary control of the sawtooth stability can be achieved by current drive just outside the $q=1$ surface. With counter-current drive the local shear at $q=1$ builds up faster, which results in shorter period sawteeth, i.e. destabilization [26].

One possibility to control sawteeth is by means of ICRF waves, which affect the sawteeth in three different ways depending on the ion cyclotron resonance layer position and on the toroidal direction of the waves (i.e. the ICRF antenna phasing). The first, as referred to earlier, is a sawtooth stabilization due to an increased fast ion-pressure in the core, when the ion cyclotron resonance layer is located in the centre $[27,28]$. The second is a possible sawtooth destabilization by ion cyclotron current drive (ICCD) as obtained with an 
antenna phasing of $+90^{\circ}$ or $-90^{\circ}$ and proper localization of the ion cyclotron resonance in the vicinity of the sawtooth inversion radius. Local magnetic shear control by ICCD has been demonstrated previously on the Joint European Torus (JET) [29], and a number of experiments have been performed recently to study ICCD in more detail and quantify its effect on sawteeth [30-32]. Finally, also the local heating from the ICRF will affect the sawteeth.

In this paper, we describe experiments performed on JET, in which we used, for the first time, ICCD produced by waves at the 2nd harmonic hydrogen resonance frequency to modify the shear on $q=1$. A summary of these results has been reported in $[11,33]$. Before going into the details of the experimental results, section 2 presents a brief review of the relevant physics concerning NTMs, sawteeth, and ICCD and fast ion generation. In section 3, the main experimental constraints and choices for experimental scenarios and methods are discussed. The experimental results are described in section 4, and discussed in the theoretical framework presented in section 2. Finally, section 5 presents a summary and provides an outlook on future implementations of the presented NTM seed island control scheme.

\section{Physics background}

\subsection{Neoclassical tearing modes}

The evolution of the width, $w$ of a NTM can be described by the generalized Rutherford equation [34,2]. For the present discussion the generalized Rutherford equation is conveniently written in the form

$\frac{\tau_{\text {res }}}{r_{\text {res }}} \frac{\mathrm{d} w}{\mathrm{~d} t}=r_{\text {res }} \Delta^{\prime}(w)+r_{\text {res }} \mathrm{C}_{\beta} \frac{\beta_{\mathrm{p}}}{w}\left(\frac{1}{1+w_{\mathrm{d}}^{2} / w^{2}}-\frac{w_{\mathrm{pol}}^{2}}{w^{2}}\right)$,

where $r_{\text {res }}$ is the minor radius of the mode resonance, and $\tau_{\text {res }} \equiv \mu_{0} r_{\text {res }}^{2} / 1.22 \eta$ is the local resistive timescale, with $\eta$ the neoclassical resistivity. The first term on the right-hand side is the classical tearing mode stability parameter $\Delta^{\prime}(w)$, which is defined as the jump across the magnetic island in the derivative of the perturbed helical flux function normalized to its value at $r_{s}$. In the remainder we restrict ourselves to $\Delta^{\prime}<0$, the case of a linearly stable tearing mode. The second term represents the instability drive from the bootstrap current perturbation, where $w_{\mathrm{d}}$ accounts for incomplete pressure flattening across the magnetic island as a consequence of a finite parallel transport timescale [35]. The third term corresponds to the effect of the ion polarization current that is the consequence of a finite island rotation with respect to the surrounding plasma [36,37]. Although, the theory on this subject is still developing (see, for example, [38,39] and references therein), here the polarization current term is taken to be stabilizing; a destabilizing contribution appears to be inconsistent with most data [5]. For the present discussion it suffices to note that both $w_{\mathrm{d}}$ and $w_{\text {pol }}$ provide a stabilizing effect at small island widths.

Typical phase diagrams of $\mathrm{d} w / \mathrm{d} t$ as a function of $w$ are sketched in figure 1 for different values of $\beta_{\mathrm{p}}$. When the plasma pressure exceeds some critical value, $\beta_{\text {crit }}$, a region of positive island growth appears between a finite minimum island size

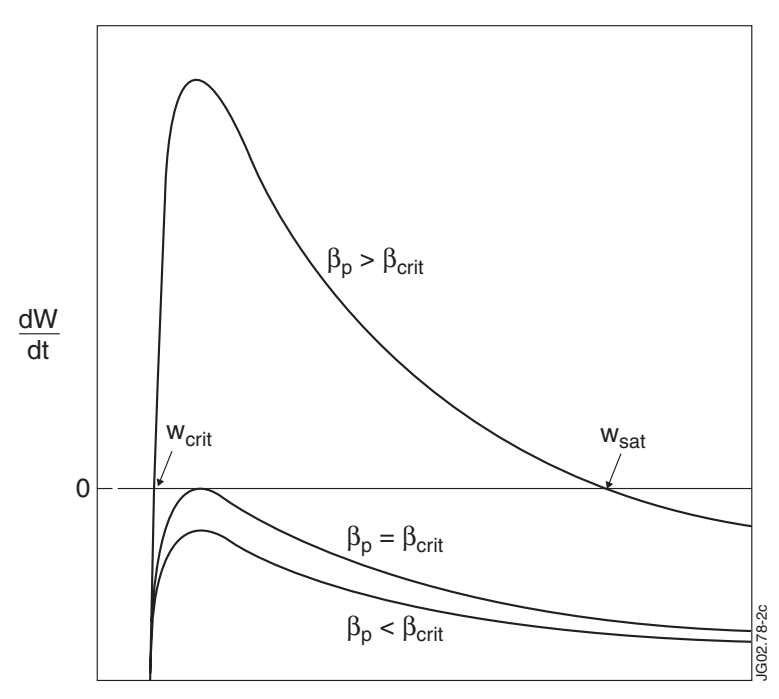

w

Figure 1. Phase diagram of a NTM, equation (1). The growth rate $\mathrm{d} w / \mathrm{d} t$ of the tearing mode magnetic island is given for three values of $\beta_{\mathrm{p}}$ : larger than, equal to, and smaller than the critical value $\beta_{\text {crit }}$. For $\beta_{\mathrm{p}}>\beta_{\text {crit }}$ the mode is meta stable.

$w_{\text {crit }}$ and the saturated island size $w_{\text {sat }}$. Apart from $\beta_{\mathrm{p}}>\beta_{\text {crit }}$, destabilization of a NTM requires some magnetic perturbation to generate a finite seed island with a size $w_{\text {seed }}$, which exceeds the critical island size $w_{\text {crit }}$. Thus, at the time of mode onset $\beta_{\mathrm{p}}$ can be well above $\beta_{\text {crit }}$, which also explains the hysteresis often observed with NTM.

The NTM seeding process itself is quite complex [12-14], and its discussion goes beyond the scope of the current paper. In case of the $m=3, n=2 \mathrm{NTM}$, the seed island is mostly seen to be generated by sawteeth (either by the pre- or post-cursor or by the sawtooth collapse itself) [40,41]. For discharges heated only by NBI, these processes result in a relatively simple scaling of the normalized pressure at the time of triggering of the NTMs, $\beta_{\text {Nonset }}$, with $\rho_{*}$ [5]. For the $m=3, n=2$ NTMs in JET the scaling of the mode onset as obtained from an extended database of NBI heated discharges is given by [41]

$$
\beta_{\text {Nonset }}=95.5 \rho_{*}^{0.71 \pm .14} \text {. }
$$

This is not a very sharp limit. Because of randomness of the necessary seeding perturbation, the mode onset in individual discharges exhibits a spread with respect to this scaling. Control or reduction of the perturbations that generate the seed, i.e. reduction of the sawtooth perturbation, is expected to result in significantly higher pressures before a NTM is triggered.

\subsection{Sawteeth}

It is well-established that strong ICRF heating can increase the period between sawtooth crashes [42-44], the accepted mechanism being that heated ions with bounce-averaged precessional drift frequencies in excess of the mode frequency have a stabilizing effect on the $m=1$ internal kink mode. However, this stabilization is most effective when the ICRF power is deposited at a major radius close to that of the magnetic axis, $R_{0}$ [27]: fast particle effects are expected to 
be much less significant when the ICRF resonance surface is close to being tangential to the $q=1$ surface.

A detailed analysis of the present experiments with 2 nd harmonic $\mathrm{H}$-minority resonance [32] has shown that, except for central ICRF resonance, the fast ion effects in these discharges are indeed unimportant. According to this analysis, the present experiments are in the 'semi-collisional' regime as identified in the sawtooth model of Porcelli et al [45]

$$
c_{\rho} \hat{\rho}>\delta \hat{W}>-\frac{1}{2} \omega_{* \mathrm{i}} \tau_{\mathrm{A}},
$$

where $\delta \hat{W}$ is a normalized measure of the $m=1$ internal kink mode energy, $\hat{\rho}$ is the thermal ion Larmor radius normalized to the $q=1$ radius $r_{1}, c_{\rho}$ is a constant of order unity, $\tau_{\mathrm{A}}=\sqrt{3} R_{0} / c_{\mathrm{A}}, c_{\mathrm{A}}$ being the Alfvén speed, and $\omega_{* \mathrm{i}}$ is the ion diamagnetic frequency at $q=1$. In this case the physics of the reconnection layer around the $q=1$ surface plays a crucial role in determining the sawtooth crash. The reconnecting mode becomes unstable when the growth rate exceeds a finite value determined by electron or ion diamagnetic effects [46], which results in a condition for the sawtooth trigger of the form $[45,47]$

$$
s_{1}>s_{\text {crit }},
$$

where $s_{1}$ is the shear at the $q=1$ surface. The critical shear, $s_{\text {crit }}$, has a strong dependence on various parameters at the $q=1$ surface, and has different expressions depending on whether the mode is in the resistive or ion-kinetic regimes. Thus, in the present experiments sawtooth destabilization is achieved by means of locally modifying the shear near $q=1$ with the help of localized ICCD [32].

\subsection{Ion cyclotron current drive}

The method to drive current by heating either minority ions with toroidally directed waves at a frequency equal to the ion cyclotron frequency, or majority ions at harmonics of the cyclotron frequency, was proposed by Fisch [48]. In the thin orbit width approximation used in his model, ion cyclotron heating and current drive is caused by diffusion of ions mainly in velocity perpendicular to the background magnetic field when the ions satisfy the resonance condition $\omega-k_{\|} v_{\|}=n \omega_{\mathrm{ci}}$. Here, $\omega$ is the wave angular frequency, $k_{\|}$is the parallel wave number, $v_{\|}$is the ion parallel velocity, $n$ is the harmonic number and $\omega_{\mathrm{ci}}$ is the cyclotron frequency of the resonating ions. Since the magnetic field is decreasing with major radius in a tokamak, the wave will resonate with particles having $v_{\|}>0\left(v_{\|}<0\right)$ on the outboard (inboard) side of the unshifted resonance $\omega=n \omega_{\mathrm{ci}}$ for $k_{\|}>0$. This gives rise to a modification of the current profile around the magnetic surface intersecting the cyclotron resonance in the midplane.

When finite orbit widths and the ICRF-induced diffusion in velocity and real space are included, new current drive mechanisms appear $[49,50]$. For example, the finite orbit widths of trapped resonating ions give rise to a current of diamagnetic type [49], while in the thin orbit approximation it is only the passing ions that can contribute to the driven current. The current density profile generated by trapped ions is always bipolar and is such that it tends to decrease the total current gradient close to the resonance surface. When ICRF power per particle is high or the resonance is on the low-field side (LFS) of the torus, ICRF-accelerated ions tend to be mainly trapped and the contribution to the driven current by trapped resonating ions becomes the dominating one.

For the present series of discharges, these effects of the ICRF have been modelled extensively with the PION [51,52] and FIDO [53] codes. The results of this modelling are described in detail in a separate paper [32]. They show that for the current experiments the dominant ICCD contribution comes from trapped energetic ions, and that the driven current density profile is indeed bipolar such that it decreases the shear in a narrow $(\sim 10 \mathrm{~cm})$ region just outside the resonance layer and increases the shear on both sides of this narrow region.

\section{Experimental constraints and methods}

Most experiments concerning NTMs on JET have been performed for toroidal magnetic fields $B_{0}$ in the range of 1.0-1.7 T, i.e. for values well below those for typical JET operations. The main reason is that the available power from NBI alone is not sufficient to achieve the parameters at which NTMs are routinely triggered for $B_{0}>1.7 \mathrm{~T}$. With this constraint on the magnetic field and the requirement for current profile tailoring by ICCD at the $q=1$ surface, ICRF waves with a frequency of $42 \mathrm{MHz}$ corresponding to the 2nd harmonic hydrogen resonance frequency, have been used, with a hydrogen minority concentration of around $10 \%$. This places the resonance layer on the high-field side (HFS) or LFS of the $q=1$ surface for magnetic fields of $1.3 \mathrm{~T}$ or $1.5 \mathrm{~T}$, respectively. To ensure similar safety factor profiles, the experiments have been carried with a fixed ratio of the toroidal magnetic field to the plasma current: $B_{0} / I_{\mathrm{p}} \approx 1 \mathrm{~T} \mathrm{MA}^{-1}$.

At these reduced field values, the transition to $\mathrm{H}$-mode already occurs for modest levels of total heating power. This means that the experiments are characterized by periods of strong ELMs activity. Under these conditions, the coupling of the ICRF waves can be severely reduced. Also, the sawteeth can become difficult to detect amongst the ELMs. Moreover, for the lower fields, central electron temperature measurements from electron cyclotron emission are either not available or in cut-off as a consequence of high densities in these discharges at the time of NTM triggering. The sawtooth evolution has been studied essentially with the central soft x-ray emission signals.

The effects of local ICCD on the sawteeth has been ascertained both in a few dedicated discharges with ICRF only performing a slow magnetic field ramp in order to move the resonance layer across the sawtooth inversion radius [30,32], as well as in an extended low-power phase in several discharges from the current set. In a typical discharge, $2.5 \mathrm{MW}$ of lowpower NBI is applied at $t=20 \mathrm{~s}$, followed by a slow ramp of ICRF power up to $5 \mathrm{MW}$ from $t=21$ to $23 \mathrm{~s}$. After a $1 \mathrm{~s}$ steady phase to determine the resulting sawtooth activity, a slow ramp up to full NBI power (17 MW) is performed to determine the plasma pressure at which the $m=3, n=2$ NTM is triggered. Such a typical discharge scenario is shown in figure 2 for JET discharge no 52083. The triggering of the NTM is diagnosed from the sudden rise of the associated $n=2$ component of the magnetic field perturbations measured at the edge by magnetic pick-up coils, and confirmed by the corresponding spectrogram. The size of the $m=3, n=2$ magnetic 


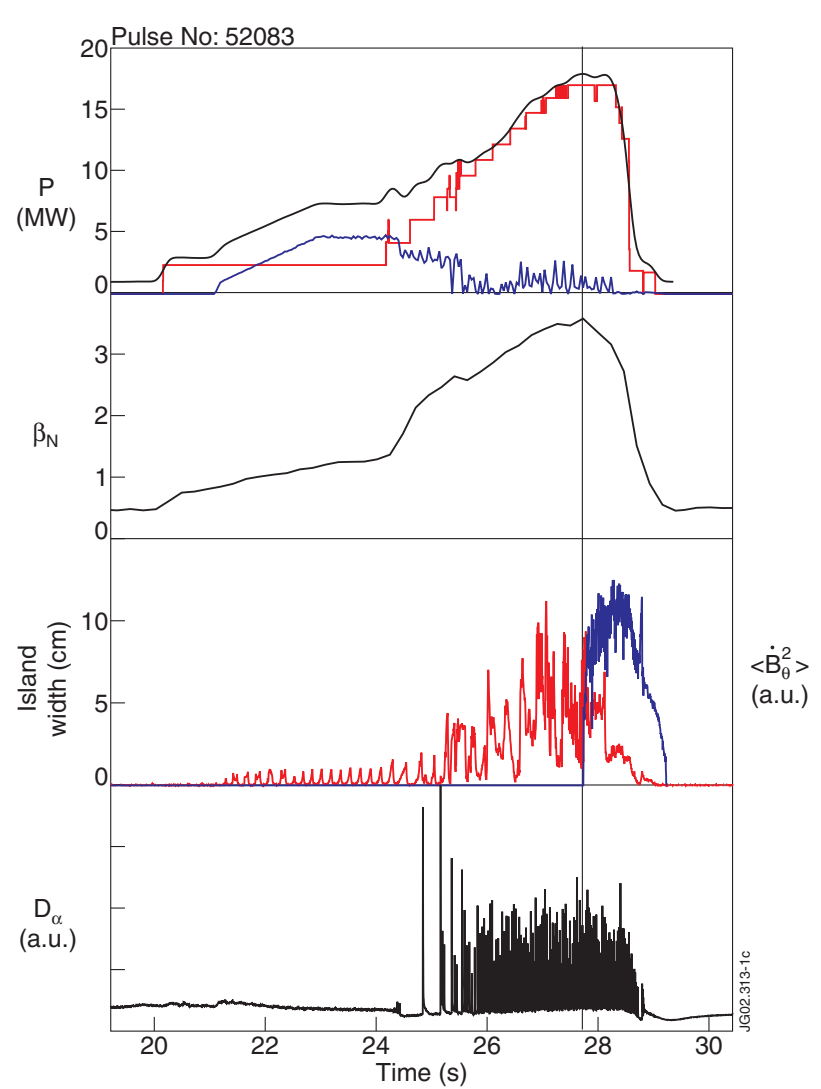

Figure 2. A discharge $\left(B_{0}=1.56 \mathrm{~T}\right.$ and $\left.I_{\mathrm{p}}=1.56 \mathrm{MA}\right)$ in which the ICRF resonance is slightly outside $R_{\text {inv }}$ on the LFS in order to destabilize sawteeth. Shown are the power waveforms (black $=$ total, red $=\mathrm{NBI}$, blue $=\mathrm{ICRF} 42 \mathrm{MHz}-90^{\circ}$ phasing), $\beta_{\mathrm{N}}$, the $n=1$ (red) component of the magnetic perturbation plus the width of the $m=3, n=2$ magnetic island (blue) as calculated from the magnetic perturbation, and the $\mathrm{D}_{\alpha}$ signal. Triggering of the $m=3, n=2 \mathrm{NTM}$ is indicated by the vertical line. Note the reduction in ICRF coupling during the high power phase due to the frequent ELMs.

island is obtained using the method as described in [9]: the magnetic perturbation measured at the edge is mapped back to the resonance surface using the cylindrical approximation, and the resulting expression is then recalibrated for the correct island size as measured by the fast ECE radiometer.

\section{Experimental results}

\subsection{ICRF sawtooth stabilization and NTM triggering}

As mentioned previously, in plasmas with significant ICRF heating the $\beta_{\mathrm{N}}$ at which an NTM is triggered is found to be well below that normally expected from plasmas with only NBI [10], except where the NBI causes a long period initial sawtooth [11]. The reason for this is the effect of the ICRF on the sawtooth behaviour: when located within the $q=1$ surface, ICRF tends to increase the central fast ion pressure which acts to stabilize the $m=1, n=1$ mode responsible for the sawtooth collapse. As a consequence longer period sawteeth are found with application of central ICRF. Such a longer period sawtooth is most likely associated with a larger magnetic perturbation, as the amount of penetrated flux during the sawtooth ramp and, consequently, reconnected flux in
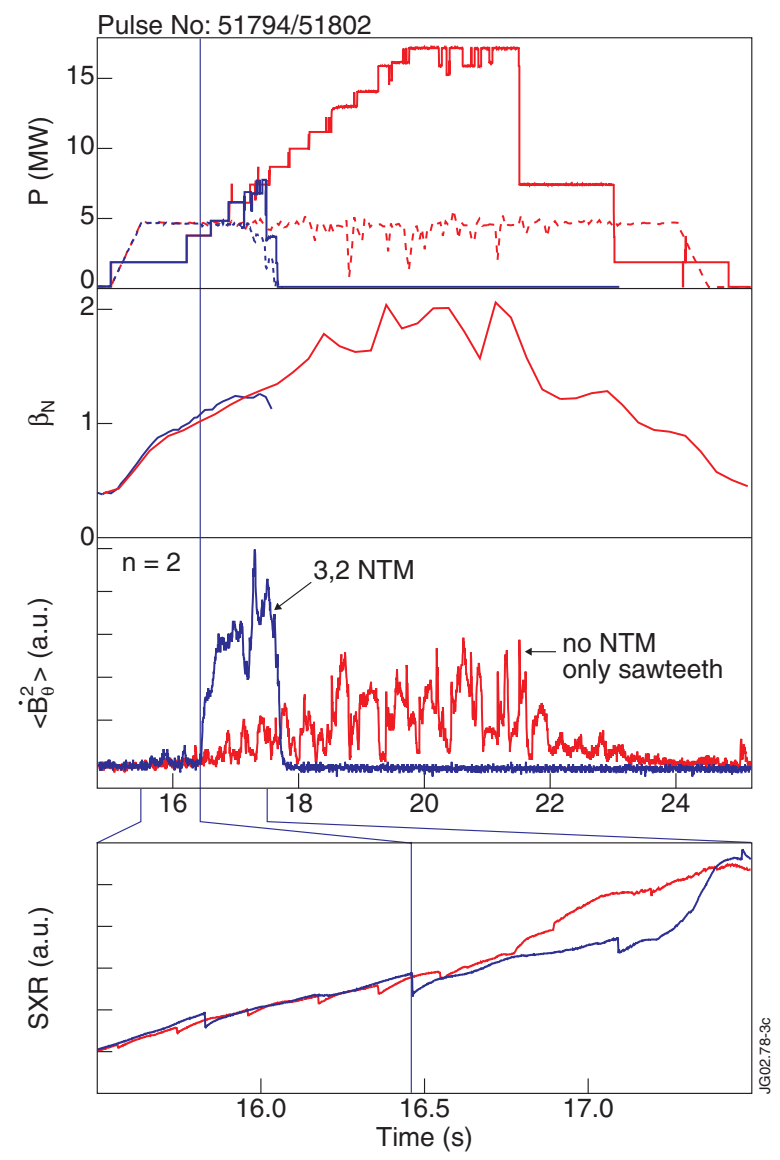

Figure 3. Discharges with $B_{0}=2.4 \mathrm{~T}, I_{\mathrm{p}}=2.4 \mathrm{MA}$, and

$\mathrm{H}$-minority ICRF at $42 \mathrm{MHz}$ (the resonance is slightly inside $R_{\text {inv }}$ on the HFS). The discharges differ only in ICRF phasing: blue $=51794$ with $+90^{\circ}$, red $=51802$ with $-90^{\circ}$. Shown are from top to bottom: NBI and ICRF power (- - - ); $\beta_{\mathrm{N}} ; n=2$ component of the magnetic perturbation. A large sawtooth in 51794 at $t=16.46 \mathrm{~s}$ triggers a $m=3, n=2$ NTM (indicated by the vertical line). The bottom panel is a blow-up of the central soft $\mathrm{x}$-ray emission showing the difference in sawtooth behaviour around this time.

the sawtooth crash is expected to increase with the sawtooth period. Thus, also the sawtooth generated seed islands are expected to be larger. The reduced rotation in case of ICRF as compared to cases with NBI only can also be a contributing factor in the generation of larger seeds [54].

The importance of the ICRF scenario and the consequences of sawtooth stabilization on NTM triggering is well illustrated by the following set of two similar discharges [33], shown in figure 3. In both discharges 51794 and 51802, $5 \mathrm{MW}$ of ICRF is applied for 1st harmonic hydrogen minority heating near the $q=1$ surface. Only the antenna phasing is different between the discharges: case of 51794, the antenna phasing is $+90^{\circ}$ in case of 51794 , and $-90^{\circ}$ in case of 51802 . As shown in previous experiments [28], with $+90^{\circ}$ phasing $\left(-90^{\circ}\right.$ phasing) the turning point of trapped resonating particles undergoes an inward (outward) drift. This leads to higher energetic ion pressure inside $q=1$ with the $+90^{\circ}$ phasing. A detailed analysis of the ICRF in these discharges by means of the PION and FIDO codes shows that the central fast ion pressure is indeed significantly larger for discharge 51794. A contributing factor to the larger fast ion density is also the longer fast ion slowing down time in this discharge as a 
consequence of the somewhat different plasma parameters. This difference in central fast ion pressure results in very different sawtooth periods as can be seen from the bottom panel of figure 3. Long period sawteeth are found in discharge 51794. The figure also shows the $n=2$ activity for both these discharges, which clearly demonstrates that in case of the long period sawteeth in discharge 51794, an NTM is triggered by the crash of a sawtooth with a period of over $600 \mathrm{~ms}$ at the relatively low value of $\beta_{\mathrm{N}}=1.2$. In discharge $51802 \mathrm{a}$ maximum $\beta_{\mathrm{N}}=2.1$ is reached, while only sawtooth activity is observed on the $n=2$ signal. In the latter case, an NTM is not triggered.

\subsection{Sawtooth control by 2 nd harmonic hydrogen resonance ICCD}

In order to demonstrate the effect of the 2 nd harmonic hydrogen minority heating and current drive on sawteeth, a number of discharges has been performed in which the magnetic field has been slowly varied during the ICRF such that the resonance layer is scanned through the sawtooth inversion radius, $R_{\text {inv }}$, on either the HFS or LFS. In figure 4 the results of discharge 51800 are shown in which $5 \mathrm{MW}$ of ICRF power has been coupled with $-90^{\circ}$ antenna phasing. The 2nd harmonic hydrogen resonance layer has been scanned through $R_{\text {inv }}$ on the LFS. Minima in the sawtooth period are found with the resonance placed on either side of $R_{\text {inv }}$, whereas a maximum sawtooth period is obtained for the resonance almost at $R_{\mathrm{inv}}$. The sawtooth period increases again as the resonance layer approaches the magnetic axis $R \approx 3 \mathrm{~m}$, which can be explained by an increased central fast ion pressure in this case. In contrast, the behaviour around $R_{\text {inv }}$ can only be understood as an effect of ICCD. This is supported by PION and FIDO modelling, which shows that the ICCD is dominated by a

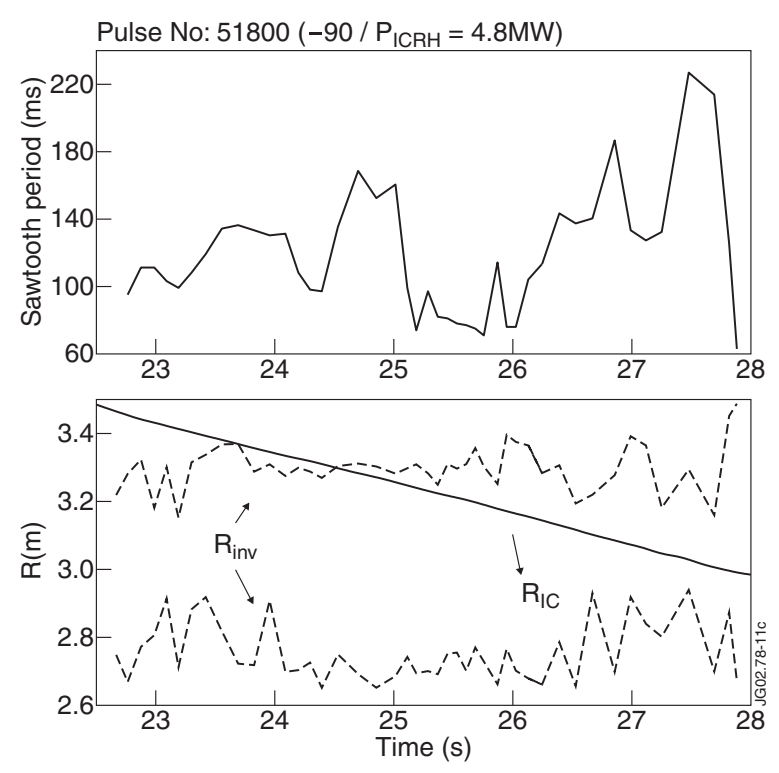

Figure 4. The evolution of the sawtooth period in discharge 51800. $5 \mathrm{MW}$ of 2 nd harmonic $\mathrm{H}$ resonance ICRF is applied with $-90^{\circ}$ phasing and $42 \mathrm{MHz}$. In the time window displayed the magnetic field and plasma current are ramped from 1.6 to $1.4 \mathrm{~T} \mathrm{MA}^{-1}$ to scan the resonance layer (-) through the sawtooth inversion radius (- - - ) on the LFS. current of the diamagnetic type caused by the finite orbit widths of trapped resonating ions resulting in a bipolar current perturbation consistent with the observed changes in sawtooth period [32]. PRETOR transport code calculations confirm that the current perturbation from ICCD is large enough to affect the sawteeth [55]. Because of its origin, this driven current is found to be not very sensitive to the ICRF antenna phasing, and similar effects on the sawtooth period are indeed obtained with $+90^{\circ}$ phasing $[30,32]$.

When the 2nd harmonic hydrogen resonance layer is placed on the HFS, sawtooth destabilization with ICRF $\left(-90^{\circ}\right.$ phasing) is again observed with the resonance layer positioned just outside $R_{\text {inv }}$. In this case the modelling shows that the resonating energetic ions are mostly passing, and the driven current and energetic ion profiles are significantly broader than for the LFS case. For a more detailed analysis of the ICRF generated non-inductive current and fast ion populations in these kind of discharges, and their effects on sawteeth the reader is referred to [32].

\subsection{NTM seed island control by sawtooth destabilization}

4.3.1. HFS resonance. A series of discharges has been performed in which the constant magnetic field has been varied on a shot-to-shot basis. In addition, a comparison of discharges with ICRF to discharges without ICRF has been made. Figure 5 shows the results for three different discharges. The magnetic field is $1.2 \mathrm{~T}$ and the plasma current is $1.2 \mathrm{MA}$. In discharge 51994 about $5 \mathrm{MW}$ of ICRF power is coupled from $t=21$ to $30 \mathrm{~s}$. The resonance layer is just outside $R_{\text {inv }}$ on the HFS, in order to destabilize sawteeth with ICCD. During the ICRF phase, the NBI is slowly ramped to full power. To establish the effect of ICCD, in discharge 51995 the identical NBI power is applied without ICCD, while in discharge 52712 the identical total power is supplied by NBI alone.

In both discharge 51995 and 52712 a $m=3, n=2$ NTM is triggered around $t=22.6 \mathrm{~s}$ by a sawtooth with a period of about $160 \mathrm{~ms}$ and $300 \mathrm{~ms}$, respectively. In case of 51995, also a large ELM only $20 \mathrm{~ms}$ after the sawtooth crash at $t=22.67$ could have contributed to the destabilization of the NTM. At the time of $m=3, n=2$ NTM onset, the normalized pressures or plasma beta are $\beta_{\text {Nonset }}=2.1$ and 2.7 in discharges 51995 and 52712, respectively. Around the same time the typical sawtooth period in discharge 51994 with ICCD just outside $q=1$ is about $130 \mathrm{~ms}$ with very similar normalized pressure to 51995 , in spite of the higher total heating power. In the latter case, a $m=3, n=2$ NTM is triggered much later at $t=26.2 \mathrm{~s}$, and at the much higher pressure of $\beta_{\mathrm{N}}=3.6$. At that time the plasma pressure is already decreasing. A maximum $\beta_{\mathrm{N}}=3.8$ had been reached before. The deterioration of confinement is caused by a $m=5$, $n=4 \mathrm{NTM}$ triggered around $t=25.0$ followed by the triggering of a $m=4, n=3 \mathrm{NTM}$ around $t=26.0$. When the plasma pressure is high, the central magnetic field is decreased by the diamagnetic effect, such that the ICRF resonance layer is moved away from its optimal position for sawtooth destabilization. However, for plasma pressures in excess of $\beta_{\mathrm{N}} \geqslant 3$, also fishbones are usually present in the plasma [40], and it is not clear whether fishbones or sawteeth have triggered these NTMs. 


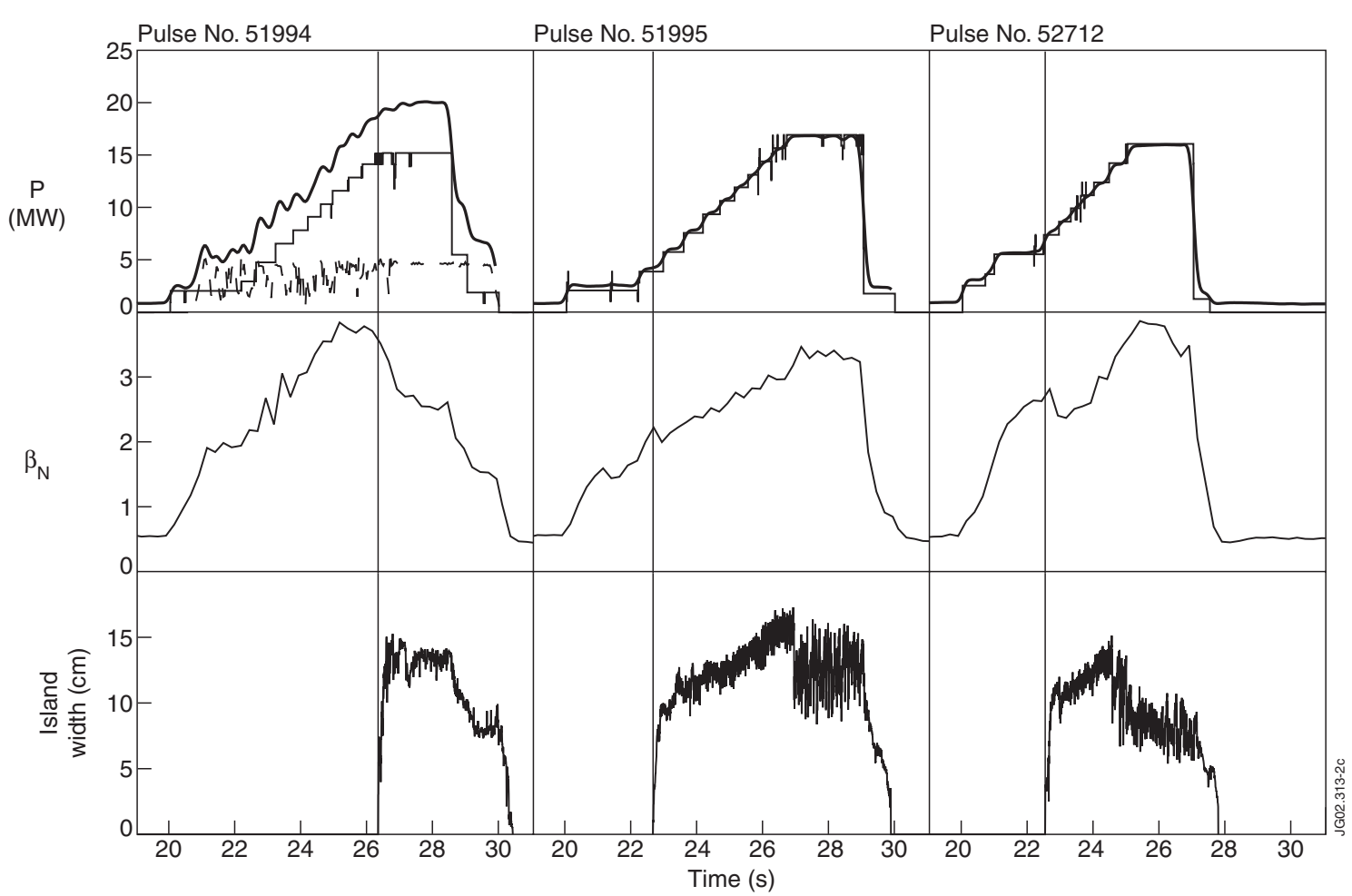

Figure 5. Results for three discharges with $B_{0}=1.20 \mathrm{~T}$ and $I_{\mathrm{p}}=1.20 \mathrm{MA}$ (from left to right: 51994, 51995, 52712). In 51994, $5 \mathrm{MW}$ of ICRF ( $42 \mathrm{MHz}$ and $-90^{\circ}$ phasing) is applied (dashed curve in top panel) for sawtooth destabilization. The panels show from top to bottom: the power wave forms (total power, NBI and ICRF power), $\beta_{\mathrm{N}}$, and the width of the $m=3, n=2$ magnetic island as calculated from the $n=2$ magnetic mode activity. The $m=3, n=2$ NTM onset is indicated by the vertical lines.

The improvement in terms of increased $\beta_{\text {Nonset }}$ that is obtained in the case with ICRF, is further illustrated by figure 6 , which shows the trajectory of the three discharges in $\beta_{\mathrm{N}}$ versus $\rho_{*}$. In the two discharges with NBI-only, the $m=3, n=2$ NTM is indeed triggered at a normalized pressure consistent with the mode onset scaling as given by equation (2), shown by the black curve. In contrast, the trajectory for discharge 51994 with ICRF significantly exceeds this pressure limit before the NTM is triggered. This improvement is attributed to the sawtooth destabilization from the ICCD just outside the inversion radius in discharge 51994. However, the sawtooth destabilization is difficult to quantify on the basis of the current data. In the comparison of the sawtooth periods at the time of NTM onset in the discharges without ICCD, it must be noted that NBI produced energetic ions can stabilize sawteeth, though less efficiently than central ICRF waves [56]. The difference in NBI power between 51994 and 52712, might be sufficient to explain the longer sawtooth period in 52712. However, the longer sawtooth period in discharge 51995 ( 160 ms) with identical NBI power demonstrates that sawteeth have been effectively destabilized by ICCD in 51994 ( $\sim 130 \mathrm{~ms})$. The comparison between discharge 51994 and both 51995 (with identical NBI) and 52712 (with identical total power) is also important as the sawtooth period is not the only parameter determining the seeding of NTM. Plasma rotation is important in the seeding process as well. In particular, smaller differential rotation between $q=1$ and $q=1.5$ is seen to lead to lower values of $\beta_{\text {Nonset }}$ [54].

That the situation is even more complex, is seen by a closer look at the evolution of these three discharges up to the

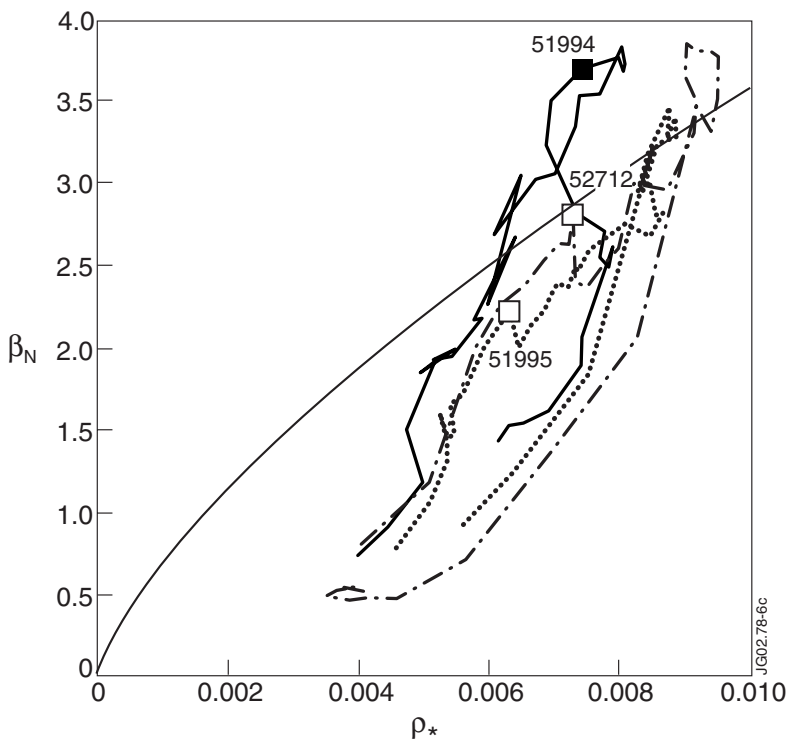

Figure 6. The evolution in terms of $\beta_{\mathrm{N}}$ and $\rho_{*}$ of discharges 51994 $(-), 51995(\cdots \cdots)$, and $52712(---)$. The squares indicate the time at which the $m=3, n=2$ NTM is triggered. The thin curve represents the scaling of the pressure limit for $m=3, n=2$ NTM onset (equation (2) as obtained from a large database of NBI-only discharges).

time of NTM onset in 51995 and 52712. Figure 7 shows the evolution during this period of the central soft x-ray emission, the $n=1$ magnetic activity, and the $\mathrm{D}_{\alpha}$ signal. For all three discharges the $\mathrm{L}$ to $\mathrm{H}$ transition occurs before $t=20.5 \mathrm{~s}$ early 


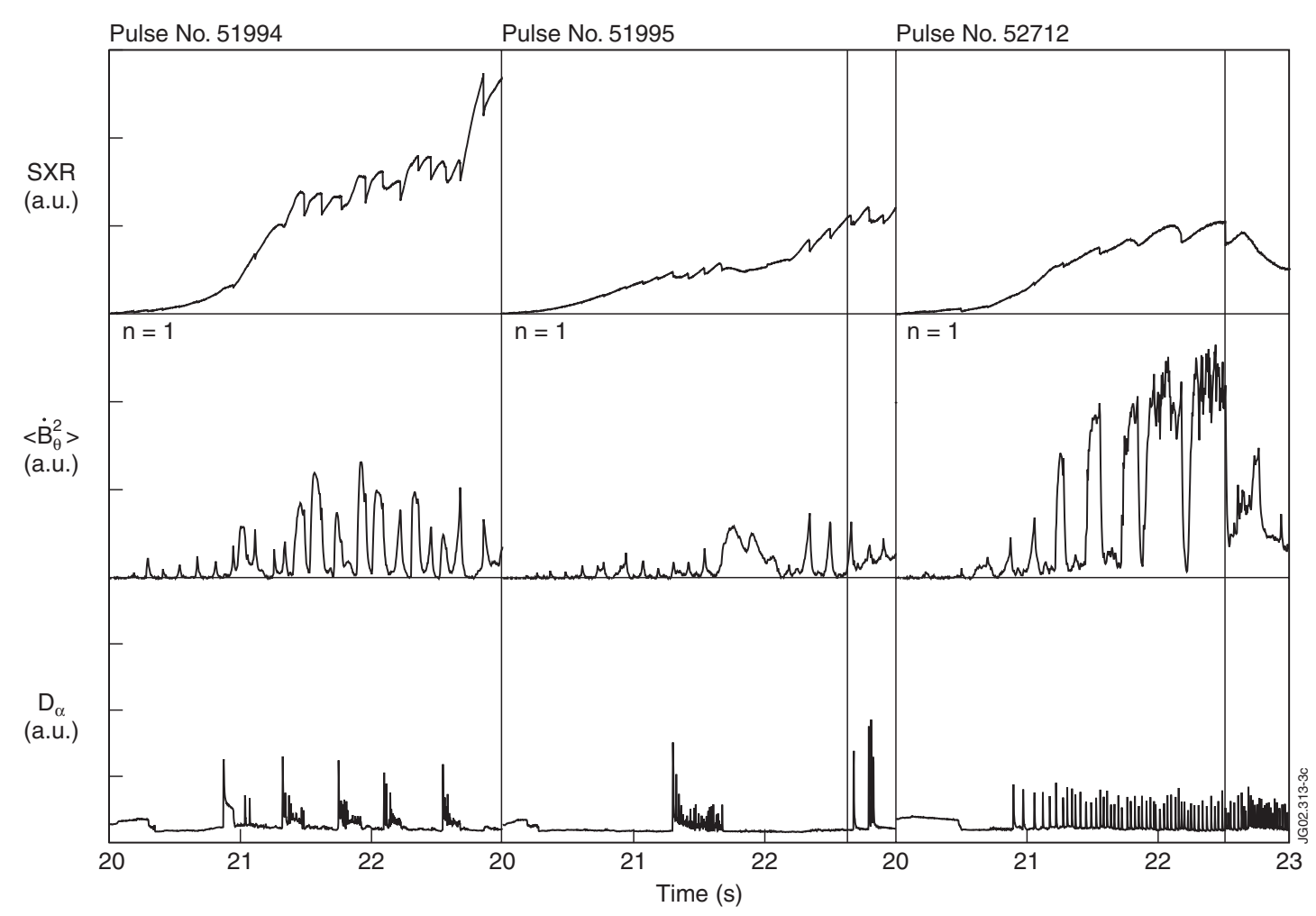

Figure 7. The central soft x-ray, $n=1$ magnetic activity, and $\mathrm{D}_{\alpha}$ for the discharges of figure 5 (from left to right: 51994, 51995, 52712) towards the time of $m=3, n=2$ NTM onset in 51995 and 51712 (indicated by the vertical lines).

in the low-power phase, but the subsequent ELM behaviour is distinctly different with apparent consequences for the confinement. Good H-mode confinement is only associated with ELM-free periods or regular type I ELMs as in 52712 with the higher NBI power. Bursts of ELMs appear to reduce the confinement in 51994 and 51995 . While in discharge 51995 the NTM is triggered after a very long ELM free period following a single initial burst of ELMs, regular bursts of ELMs continue throughout the same period in discharge 51994. This explains the relatively low $\beta_{\mathrm{N}}$ during this period in 51994, in spite of the higher total heating power. The bursts of ELMs are also likely to have slowed down the plasma rotation: in discharge 51994 the frequency of the $n=1$ sawtooth precursor between $t=22$ and $23 \mathrm{~s}$ is typically $4 \mathrm{kHz}$, while the frequency in both NBI only discharges is higher at about $7-8 \mathrm{kHz}$. Charge exchange measurements of the plasma rotation confirm that at that time the rotation in the two NBI only discharges is very similar, in spite of the difference in NBI power at the time, but is significantly larger than in discharge 51994. A spectral analysis, figure 8 , also shows that the $n=1$ oscillations in 52712 are made up of a combination of fishbones (at about $8 \mathrm{kHz}$ ) and the sawtooth precursor (at about $7 \mathrm{kHz}$ ).

4.3.2. LFS resonance. Table 1 summarizes the results of a series of discharges, in which the 2 nd harmonic $\mathrm{H}$ resonance is located mostly close to $R_{\mathrm{inv}}$ on the LFS, i.e. for magnetic fields in the range of 1.48-1.62 T. In most of these discharges about $5 \mathrm{MW}$ of ICRF power is coupled, and after an extended low-power phase the NBI is slowly ramped to full power. The sawtooth period during the extended low-power phase is given. In most cases, the $\mathrm{L}$ to $\mathrm{H}$ transition occurs early in the

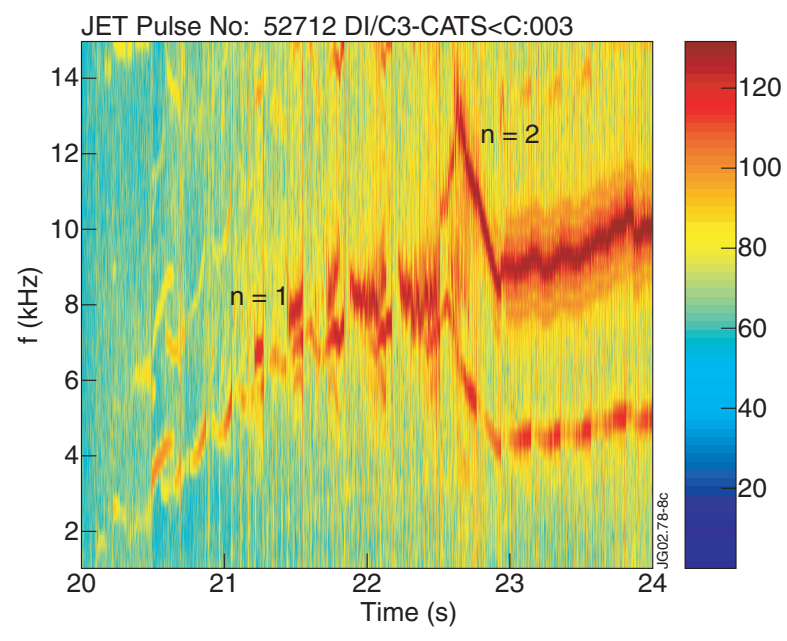

Figure 8. Spectral analysis of the signal from magnetic pick-up coils in discharge 52712 for the period leading up to and just after the triggering of a $m=3, n=2$ NTM.

NBI power ramp, and the frequent ELM activity makes the identification of individual sawteeth difficult, in particular at the time of triggering of the NTM. In agreement with the data from the magnetic field scans (like discharge 51800, figure 4), the table shows that by positioning of the 2nd harmonic $\mathrm{H}$ resonance layer $R_{\mathrm{IC}}$ somewhat outside the sawtooth inversion radius on either the HFS (52077) or LFS, the ICCD results in shorter period sawteeth. The value of $\beta_{\text {Nonset }}$ is seen to correlate even better with $R_{\mathrm{IC}}$. The highest normalized pressure at $m=3, n=2$ NTM onset $\left(\beta_{\text {Nonset }}=3.60\right)$ and shortest sawtooth period are found in discharge 52083 with $R_{\mathrm{IC}}$ 
Table 1. LFS ICCD scan. The columns present the discharge number, vacuum toroidal field, position 2 nd harmonic hydrogen cyclotron resonance $R_{\mathrm{IC}}$, and sawtooth period $\tau_{\mathrm{st}}$ all near $t=23.5 \mathrm{~s}$, and the time of onset of a $m=3, n=2$ NTM and the normalized pressure at that time. For discharge $52084 t_{\mathrm{NTM}}$ refers to the time of triggering of a $m=2, n=1$ mode (the $m=3, n=2$ mode is never triggered in this discharge).

\begin{tabular}{llllll}
\hline $\begin{array}{l}\text { Discharge } \\
\text { JET }\end{array}$ & $\begin{array}{l}B_{0} \\
(\mathrm{~T})\end{array}$ & $\begin{array}{l}R_{\mathrm{IC}} \\
(\mathrm{m})\end{array}$ & $\begin{array}{l}\tau_{\text {st }} \\
(\mathrm{ms})\end{array}$ & $\begin{array}{l}t_{\mathrm{NTM}} \\
(\mathrm{s})\end{array}$ & $\beta_{\text {Nonset }}$ \\
\hline 52077 & 1.29 & 2.78 & 214 & 24.90 & 2.55 \\
$52054^{*}$ & 1.38 & 3.02 & $300^{*}$ & 24.66 & 1.55 \\
52079 & 1.47 & 3.21 & 307 & 25.50 & 2.35 \\
52080 & 1.50 & 3.29 & 272 & 27.60 & 3.35 \\
52082 & 1.53 & 3.34 & 253 & 28.10 & 3.45 \\
52083 & 1.56 & 3.42 & 180 & 28.10 & 3.60 \\
52087 & 1.61 & 3.55 & 231 & 26.37 & 2.65 \\
52084 & 1.56 & - & 297 & 25.50 & 3.00 \\
52703 & 1.56 & - & 315 & 27.37 & 3.35 \\
\hline
\end{tabular}

* Discharge 52054 has only additional heating from central ICRF. It exhibits compound sawteeth and the period given applies to the period between full collapses; a partial collapse is seen about midway the sawtooth ramp.

somewhat outside the sawtooth inversion radius on the LFS. The evolution of this discharge has been shown in figure 2 .

However, the data do not show a straightforward correlation between the sawtooth period in the low-power phase and $\beta_{\text {Nonset }}$ for the $m=3, n=2$ NTM. Even though the sawtooth periods in the NBI-only case are larger than most of the cases with ICRF, they nevertheless show a normalized pressure at mode onset of about $\beta_{\text {Nonset }} \approx 3.0-3.3$, significantly higher than several of the ICRF cases with shorter sawtooth periods in the low-power phase. Several effects might contribute to this result. First, due to increased ELM activity during the NBI power ramp the ICRF coupling in most discharges is significantly decreased, such that sawtooth destabilization and its beneficial effect on the prevention of NTM onset might be lost. Second, plasma rotation is reduced when NBI power is replaced by ICRF power, which in the absence of effective ICCD sawtooth destabilization results in a reduced threshold for NTM onset [54]. Moreover, during the power ramp the increase in plasma pressure leads to a shift of the cyclotron resonance to a smaller major radius as a consequence of diamagnetism. This is displayed in figure 9 for the ICRF discharges of table 1 . For the LFS cases the result is a shift of the 2nd harmonic hydrogen cyclotron resonance from just outside to a position on top of $R_{\text {inv }}$ or just inside. When this happens, the effect on sawteeth can change from destabilizing to stabilizing. Figure 9 shows that the ICRF discharges can be divided into three groups. One group is characterized by a relatively high $\beta_{\text {Nonset }}$ at or above the values for the two NBI-only discharges. In these discharges the ICRF resonance comes close to the inversion radius at a late stage in the NBI power ramp. A second group is seen with $\beta_{\text {Nonset }}$ around 2.4, well below the value for the two NBI-only discharges. In these cases the ICRF resonance moves inside the inversion radius early in the power ramp, or it remains relatively far outside the inversion radius (52087). Finally, the discharge with ICRFonly has by far the lowest pressure threshold.

As the plasma parameters can vary significantly between the different discharges, the $\beta_{\text {Nonset }}$ values are better compared to the values expected from the scaling of the mode onset in

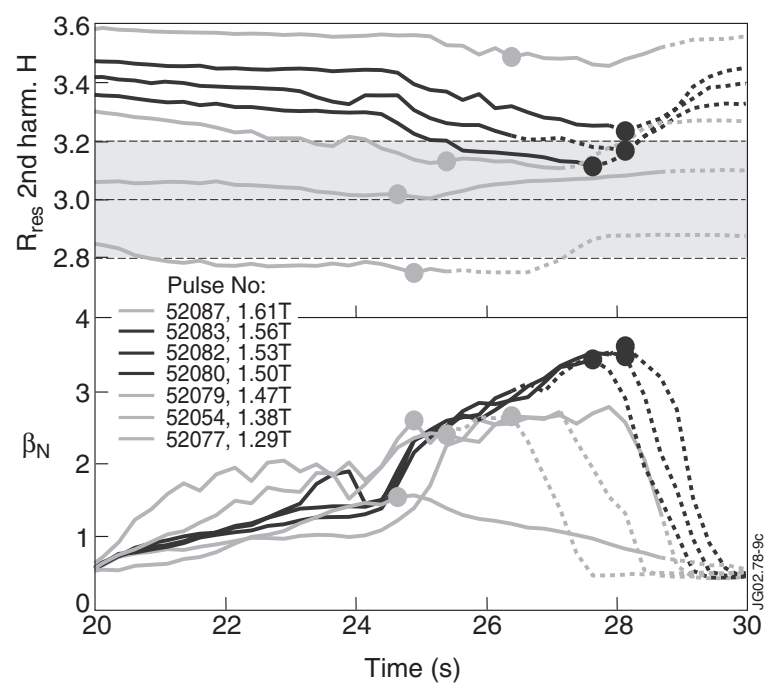

Figure 9. The evolution of the 2 nd harmonic $\mathrm{H}$ cyclotron resonance position and $\beta_{\mathrm{N}}$ for the discharges of table 1 . The dots mark the NTM onset and the dashed parts of the curves indicate when $P_{\mathrm{ICRF}}<1 \mathrm{MW}$ due to bad coupling. The shaded area represents the region inside $R_{\text {inv }}$. Reprinted from [33].

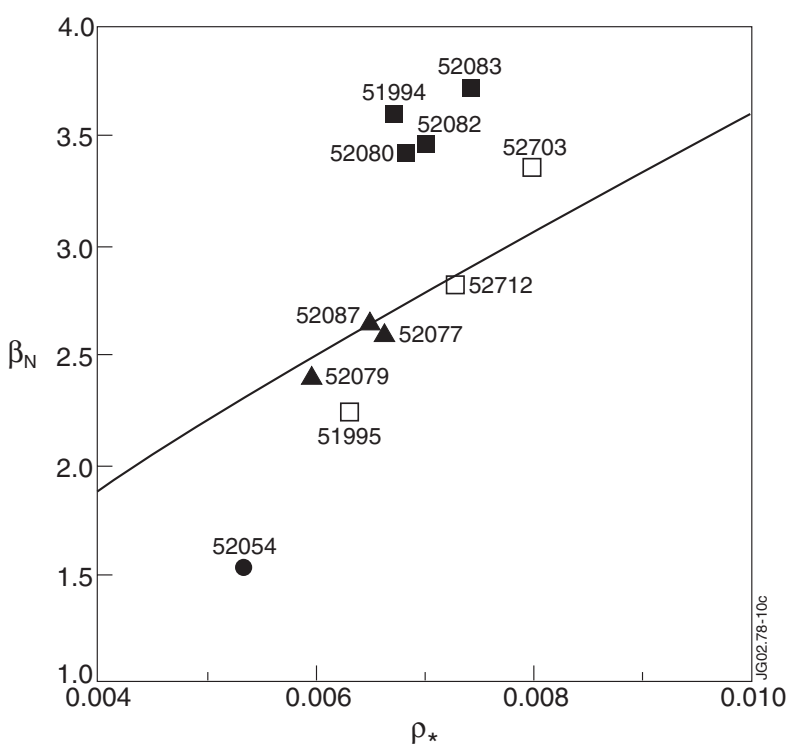

Figure 10. The normalized pressure $\beta_{\mathrm{N}}$ at $m=3, n=2$ NTM onset for all discharges from the present set of experiments. The curve represents the scaling of the pressure limit for mode onset (equation (2) as obtained from a large database of NBI-only discharges).

NBI-only discharges as given in equation (2). The $\beta_{\text {Nonset }}$ for all discharges from the present set of experiments are shown in figure 10 as a function of $\rho_{*}$. The open squares in figure 10 refer to the NBI-only cases. These are indeed found to be reasonably close to this scaling of the mode onset. Again the ICRF discharges divide into three groups. The black squares show the cases in which the pressure significantly exceeded the limit for mode onset. These include discharge 51994 and the three best LFS discharges. The black triangles refer to the second group of ICRF discharges with $\beta_{\text {Nonset }}$ around 2.4. As becomes clear from this figure the $\beta_{\text {Nonset }}$ for these discharges 
is actually close to the scaling for NBI-only, such that one is led to conclude that in these cases the ICRF had no net effect. The black circle, finally, shows the result for the discharge with central ICRF: clearly, the sawtooth stabilization in this case has led to a mode onset well below the scaling for NBI discharges.

In spite of the complex differences between individual discharges, one overriding conclusion remains: discharges with significant ICRF generally have a low threshold for triggering of NTM. However, when the resonance position of the 2nd harmonic hydrogen cyclotron resonance has been carefully chosen to destabilize sawteeth, the $\beta_{\text {Nonset }}$ is increased significantly over its value with NBI-only. Sawtooth destabilization is obtained with the ICRF resonance just outside $R_{\text {inv }}$, i.e. for only a narrow range of magnetic fields of about $0.1 \mathrm{~T}$ in correspondence with a radial width of about $20 \mathrm{~cm}$. This agrees with the results presented above of discharge 51800 (figure 4) concerning the effect of ICCD on the sawtooth period $[32,30]$.

\section{Summary and outlook}

A new strategy for the control or, more appropriately, the avoidance of NTMs is presented: by destabilizing the sawtooth the size of the NTM seed islands associated with sawteeth is reduced and higher plasma pressures are reached before NTM onset. This strategy has been explored in a series of experiments on the JET operated under the European Fusion Development Agreement (EFDA). Localized ion cyclotron current drive, produced by ICRF waves tuned to the 2nd harmonic minority hydrogen cyclotron frequency, has been used for sawtooth control. The results clearly demonstrate, how by reducing the sawtooth period with localized ICCD the pressure threshold, $\beta_{\text {Nonset }}$, for NTM onset is increased.

On the other hand, ICRF is well known to lead to sawtooth stabilization by the generation of energetic ions in the centre of the discharge. When this occurs and long period sawteeth are produced, the experiments show that $m=3, n=2$ NTMs can be triggered by these sawteeth at normalized pressures almost equal to or only slightly above the critical value for NTM stability, $\beta_{\text {crit }}$. This explains the generally lower $\beta_{\text {Nonset }}$ in discharges with significant ICRF as compared to discharges with additional heating by NBI only [10]. A contributing factor is the smaller rotation in plasmas with ICRF as compared to plasmas with NBI-only [54]. For discharges with $B_{0} \approx 2.4-2.8 \mathrm{~T}, \beta_{\text {Nonset }}$ is found to rapidly decrease to $\beta_{\text {crit }}$ as soon as the sawtooth period exceeds about $600 \mathrm{~ms}[11,33]$. In standard ELMy H-mode JET plasmas, the normalized critical pressure for $m=3, n=2$ NTMs is relatively low: $\beta_{\mathrm{Ncrit}} \approx 0.5-1.0$. It is only by a careful choice of the ICRF resonance position and phasing, such as to destabilize the sawteeth, that these adverse effects of ICRF with respect to NTMs are overcome, and that the $\beta_{\text {Nonset }}$ is increased above its value with NBI-only.

The maximum pressures reached in the present discharges with ICCD for sawtooth destabilization are very close to the ideal beta limit, $\beta_{\mathrm{N}} \approx 4$, and significantly higher than the typical $\beta_{\mathrm{N}}$ in a standard ELMy H-mode scenario for a tokamak reactor like ITER [8]. Still, these experiments have been hampered by difficulties in ICRF coupling due to ELMs (see figures 2 and 5), and by the shift of the ICRF resonance due to the diamagnetic effect as the plasma pressure is increased. Further improvements are expected when the ICRF coupling is improved and the vacuum toroidal magnetic field is controlled in order to compensate for the diamagnetic effect.

In a reactor, sawteeth have the added benefit of preventing accumulation of impurities and He-ash in the centre. Short period, small amplitude sawteeth might perform this essential task without the large fusion power excursions that would be associated with large amplitude sawteeth. However, the sawtooth stabilization coming from the energetic alpha particles are likely to make large amplitude monster sawteeth the natural mode of operation in burning plasmas [45]. Hence, the presented method of NTM control in combination with a standard ELMy $\mathrm{H}$-mode might provide an attractive scenario for burning plasmas. It could both provide stability to NTMs by control of the seed island size as well as limit central He-ash accumulation by active destabilization of frequent, small amplitude sawteeth. Within such a scenario, the task of sawtooth destabilization could also be performed by ECCD instead of ICCD. ECCD potentially provides a more localized current perturbation, and a possible steering capability of the beam launchers gives additional flexibility and control over the position of ECCD.

\section{Acknowledgments}

We are grateful to the UKAEA for the efficient operation of the JET tokamak. This research was performed under the European Fusion Development Agreement with financial support from EURATOM and in part from NWO (Nederlanse Organisatie voor Wetenschappelijk Onderzoek), the Swiss National Science Foundation, the UK Department of Trade and Industry, and the Fundacao para a Ciencia e Tecnology (FCT), Portugal.

\section{References}

[1] Chang Z. et al 1995 Phys. Rev. Lett. 744663

[2] Sauter O., LaHaye R.J., Chang Z. and Gates D.A. 1997 Phys. Plasmas 41654

[3] Hegna C.C. 1998 Phys. Plasmas 51767

[4] JET Team (prepared by G.T.A. Huysmans) Observation of neoclassical tearing modes in JET 1999 Nucl. Fusion 39 1965

[5] Buttery R.J. et al 2000 Plasma Phys. Control. Fusion 422599

[6] Koslowski H.R. et al 2000 Nucl. Fusion 40821

[7] Günter S. et al 1998 Nucl. Fusion 381431

[8] ITER Physics Basis 1999 Nucl. Fusion 392137

[9] LaHaye R.J. et al 2000 Phys. Plasmas 73349

[10] Hender T.C. et al 2001 Proc. 18th Int. Conf. on Fusion Energy (Sorrento, 2000) (Vienna: IAEA) CD-ROM file EXP3/02 http://www.iaea.org/programmes/ripc/physics/fec2000/ $\mathrm{html} /$ node1.htm

[11] Sauter O. et al 2001 28th EPS Conf. Contr. Fusion and Plasma Phys. (Funchal, Madeira, 18-22 June 2001) vol 25A (ECA) (Geneva: European Physical Society) CD-Rom Paper OR.08, http://epsppd.epfl.ch/Madeira/html/pdf/OR.08.pdf

[12] Nave M.F.F. et al 2000 27th EPS Conf. Contr. Fusion and Plasma Phys. (Budapest, 12-16 June 2000) vol 24B (ECA) p 1100

[13] Nave M.F.F. et al 2001 Triggering of neo-classical tearing modes by mode coupling Nucl. Fusion submitted

[14] Hegna C.C., Callen J.D. and LaHaye R.J. 1999 Phys. Plasmas 6130 
[15] Morris A.W. 1992 Plasma Phys. Control. Fusion 341871

[16] Zohm H. 1997 Phys. Plasmas 43433

[17] Yu Q., Günter S., Giruzzi G., Lackner K. and Zabiego M. 2000 Phys. Plasmas 7312

[18] Gantenbein G. et al 2000 Phys. Rev. Lett. 851242

[19] LaHaye R.J. et al 2001 28th EPS Conf. Contr. Fusion and Plasma Phys. (Funchal, Madeira, 18-22 June 2001) vol 25A (ECA) (Geneva: European Physical Society) CD-Rom Paper P4.007, http://epsppd.epfl.ch/Madeira/html/ pdf/P4.007.pdf

[20] Isayama A. et al 2000 Plasma Phys. Control. Fusion 42 L37

[21] Westerhof E. 1990 Nucl. Fusion 301143

[22] Pletzer A. and Perkins F.W. 1999 Phys. Plasmas 61589

[23] Warrick C.D. et al 2000 Phys. Rev. Lett. 85574

[24] Yu Q., Günter S. and Lackner K. 2000 Phys. Rev. Lett. 852949

[25] LaHaye R.J. et al 2000 Nucl. Fusion 4053

[26] Sauter O. et al 2001 Phys. Plasmas 82199

[27] Porcelli F. 1991 Plasma Phys. Control. Fusion 331601

[28] Eriksson L.G. et al 1998 Phys. Rev. Lett. 811231

[29] Bhatnagar V.P. et al 1994 Nucl. Fusion 341579

[30] Mayoral M.-L. et al 2001 Proc. 14th Topical Conf. RF Power in Plasmas (Oxnard (USA), 7-9 May 2001) AIP Conf. Proc. vol 595, p 106

[31] Nguyen F. et al 2001 28th EPS Conf. Contr. Fusion and Plasma Phys. (Funchal, Madeira, 18-22 June 2001) vol 25A (ECA) (Geneva: European Physical Society) CD-Rom Paper P2.081, http://epsppd.epfl.ch/Madeira/html/ pdf/P2.081.pdf

[32] Mantsinen M. et al 2002 Plasma Phys. Control. Fusion 44 1521

[33] Sauter O. et al 2002 Phys. Rev. Lett. 88105001

[34] Rutherford P.H. 1973 Phys. Fluids 161903

[35] Fitzpatrick R. 1995 Phys. Plasmas 2825

[36] Smolyakov A.I., Hirose A., Lazzaro E., Re G.B. and Callen J.D. 1995 Phys. Plasmas 21581

[37] Wilson H.R., Connor J.W., Hastie R.J. and Hegna C.C. 1996 Phys. Plasmas 3248

[38] Wilson H.R. et al 2001 Proc. 18th Int. Conf. Fusion Energy (Sorrento, 2000) (Vienna: IAEA) CD-ROM file
TH3/5, http://www.iaea.org/programmes/ripc/physics/ fec2000/html/node1.htm.

[39] Connor J.W., Waelbroeck F.L. and Wilson H.R. 2001 Phys. Plasmas 82835

[40] Belo P. et al 2001 28th EPS Conf. Control. Fusion and Plasma Phys. (Funchal, Madeira, 18-22 June 2001) vol 25A (ECA) (Geneva: European Physical Society) CD-Rom Paper P5.004, http://epsppd.epfl.ch/Madeira/html/pdf/P5.004.pdf

[41] Buttery R.J. et al 2002 Onset of Neoclassical Tearing Modes on JET Nucl. Fusion submitted

[42] Campbell D.J. et al 1988 Phys. Rev. Lett. 602148

[43] McClements K.G. et al 1995 Phys. Plasmas 82994

[44] Graves J.P. et al 2000 Phys. Rev. Lett. 841204

[45] Porcelli F., Boucher D. and Rosenbluth M.N. 1996 Plasma Phys. Control. Fusion 382163

[46] Sauter O. et al 1999 Theory of fusion plasmas ed J.W. Connor, E. Sindoni and J. Vaclavik (Bologna: SIF) p 403

[47] Levinton F.M. et al 1994 Phys. Rev. Lett. 722895

[48] Fisch N.J. 1981 Nucl. Fusion 2115

[49] Hellsten T., Carlsson J. and Eriksson L.-G. 1995 Phys. Rev. Lett. 743612

[50] Carlsson J., Hellsten T. and Hedin J. 1998 Phys. Plasmas 5 2885

[51] Eriksson L.-G., Hellsten T. and Willén U. 1993 Nucl. Fusion 331037

[52] Eriksson L.-G. and Hellsten T. 1995 Phys. Scr. 5570

[53] Carlsson J., Eriksson L.-G. and Hellsten T. 1994 Proc. Joint Varenna-Lausanne Int. Workshop on Theory of Fusion Plasmas (Varenna, 1994) (Bologna: Editrice Compositori) p 351

[54] Buttery R.J. et al 2001 28th EPS Conf. Control. Fusion and Plasma Phys. (Funchal, Madeira, 18-22 June 2001) vol 25A (ECA) (Geneva: European Physical Society) CD-Rom Paper P5.011, http://epsppd.epfl.ch/Madeira/html/pdf/ P5.011.pdf

[55] Angioni C. 2001 CRPP, EPFL, Lausanne, Switzerland, Private communication

[56] Angioni C. et al 2002 Plasma Phys. Control. Fusion 44205 